\title{
A wideband four-way power divider/combiner based on substrate integrated waveguide and double-layer finline
}

\author{
Yihong Zhou ${ }^{1 \text { a) }}$, Haiyang Wang ${ }^{1}$, Jiayin $\mathbf{L i}^{1}$, and Haiyan $\mathbf{J i n}^{2}$ \\ ${ }^{1}$ School of Physical Electronics, University of Electronic Science and Technology \\ of China, No. 4 Section 2 North Jianshe Road, Chengdu, 610054, China \\ ${ }^{2}$ School of Communication and Information Engineering, \\ University of Electronic Science and Technology of China, \\ No. 4 Section 2 North Jianshe Road, Chengdu, 611731, China \\ a)zhouyh@uestc.edu.cn
}

Abstract: A novel wideband four-way divider/combiner based on substrate integrated waveguide (SIW) and double-layer finline is presented. This divider/combiner designed at $29-40 \mathrm{GHz}$ is mainly composed of the doublelayer finline transition from waveguide to SIW and the double-microstrip transition from SIW to microstrip. These transitions feature the function of power divider/combiner, as well as the function of transition. The measured power-combining efficiency of this circuit at $35.6 \mathrm{GHz}$ is $86 \%$, and the combining efficiency is higher than $75 \%$ at $29-40 \mathrm{GHz}$.

Keywords: SIW, power divider/combiner, double-layer finline

Classification: Microwave and millimeter wave devices, circuits, and systems

\section{References}

[1] Y. H. Zhou, J. Y. Li, H. Y. Jin and H. Y. Wang: IEICE Trans. Electron. E93-C (2010) 703. DOI:10.1587/transele.E93.C.703

[2] Y. H. Zhou, J. Y. Li, H. Y. Jin and H. Y. Wang: IEICE Trans. Electron. E93-C (2010) 1484. DOI:10.1587/transele.E93.C.1484

[3] Y. H. Zhou, J. Y. Li, H. Y. Jin and H. Y. Wang: IEICE Trans. Electron. E93-C (2010) 1648. DOI:10.1587/transele.E93.C.1648

[4] L. Boccia, A. Emanuele, E. Arnieri, A. Shamsafar and G. Amendola: EUCAP Antenn. Propag. (2012) 3631. DOI:10.1109/EuCAP.2012.6206405

[5] H. Y. Jin and G. J. Wen: IEEE Microw. Wireless Compon. Lett. 18 (2008) 515. DOI:10.1109/LMWC.2008.2001007

[6] D. Deslandes and K. Wu: IEEE Microw. Wireless Compon. Lett. 11 (2001) 68. DOI:10.1109/7260.914305

[7] H. Y. Jin, J. Li and G. J. Wen: IEICE Trans. Electron. E93-C (2010) 205. DOI: $10.1587 /$ transele.E93.C.205

8] J. Li, G. Wen and F. Xiao: Electron. Lett. 46 (2010) 223. DOI:10.1049/el.2010. 2518 
[9] H. Nam, T. S. Yun, K. B. Kim, K. C. Yoon and J. C. Lee: APMC Microw. Conf. Proc. (2005) 4. DOI:10.1109/APMC.2005.1606310

[10] Z. Liu and G. B. Xiao: APMC Microw. Conf. Proc. (2013) 948. DOI:10.1109/ APMC.2013.6694984

\section{Introduction}

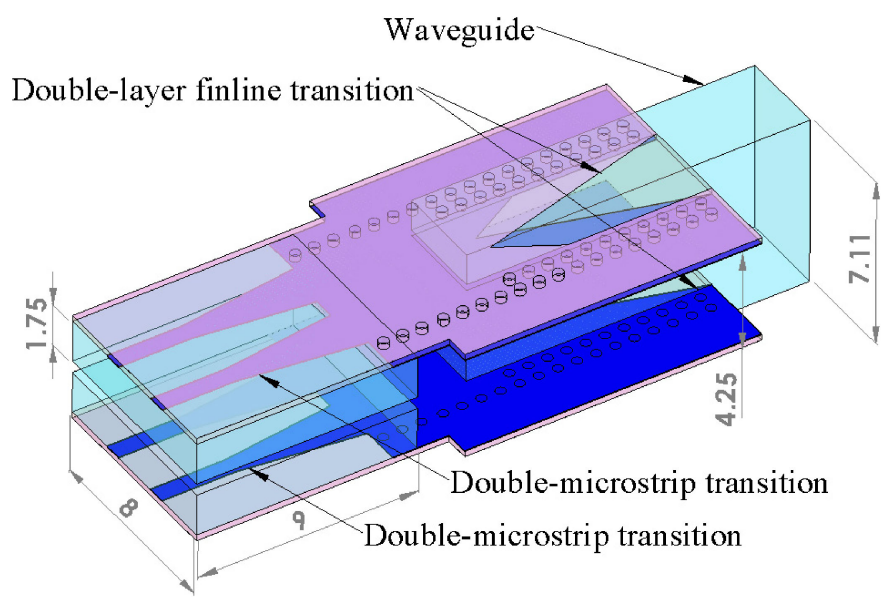

Fig. 1. Configuration of the four-way power divider/combiner based on SIW and double-layer finline

With the rapid advancements of modern communication systems, the demand for high-power solid-state power amplifiers has greatly increased. Due to the low power level of an individual solid-state device, it is necessary to combine power from multiple devices to obtain the desired power level. Some power-combining technologies based on finline and substrate integrated waveguide (SIW) have been proposed [1, 2, 3, 4, 5, 6]. In [1, 2, 3], the power combiners based on finline and double-layer finline have been proposed. These combining structures can be fabricated using the standard printed circuit board (PCB) process and easily assembled into three-dimension waveguide structure. SIW has been a popular transmission in recent years $[4,5,6,7]$, which can offer a compact and costeffective solution for integrating microwave circuits. Some combiners based on SIW have been proposed [4, 5]. In [4], a printed horn combiner/divider is presented. In [5], a four-way combiner based on half model SIW is proposed.

In this paper, based on SIW and double-layer finline, a novel wideband fourway power divider/combiner designed at $29-40 \mathrm{GHz}$ is presented, as shown in Fig. 1. In this design, the divider/combiner mainly consists of two novel transitions. One is the double-layer finline transition from waveguide to SIW, and another one is the double-microstrip transition from SIW to microstrip. The two transitions feature the function of power divider/combiner, as well as the function of transition between two different transmission lines. Since these transitions are accompanied with power division/combination, the overall structure is very compact. In addition, the precise structure based on SIW and finline can be fabricated by using the standard PCB process, and the fabrication of the overall 
divider/combiner is relatively easy. Due to the high symmetry of double-layer finline and SIW, the two transitions have essentially the characteristic of wide bandwidth. The simulated and measured results of the proposed power divider/ combiner are presented.

\section{Transition design}

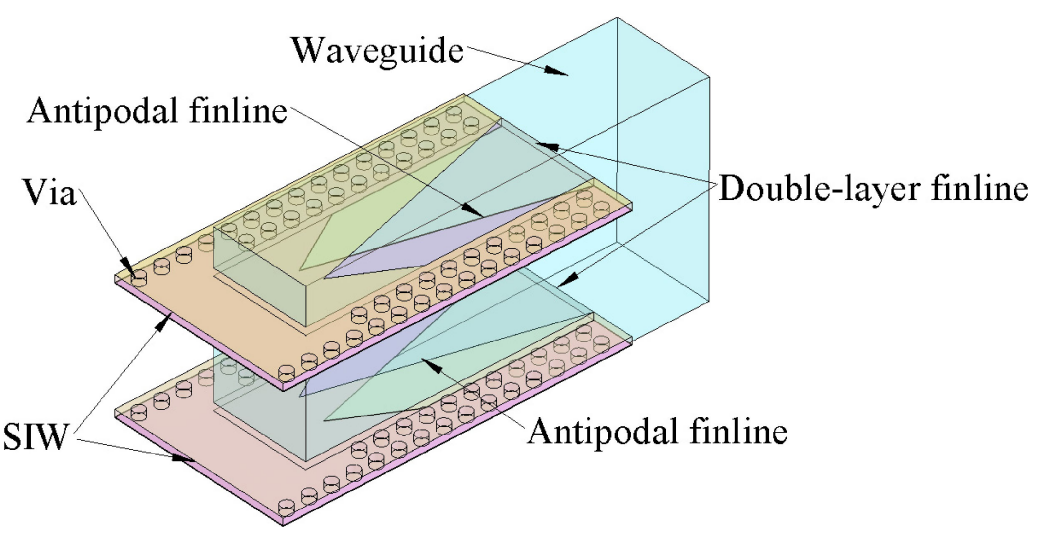

Fig. 2. Double-layer finline transition

\subsection{Double-layer finline transition design}

In [8], a broadband transition based on finline probe has also been proposed. For this transition, a cosine square function curve and a circular curve are employed to improve impedance matching between waveguide and SIW. For the sake of simplicity, a two-stage linearly tapered antipodal finline instead of the complex function curve is adopted in the proposed transition (see Fig. 2), which can translate the TE10 mode of the rectangular waveguide to the TE10-like mode of the substrate integrated waveguide by rotating the electric field.

In the previous design [2], the double-layer finline is introduced to double the branch number of the divider/combiner. In this proposed transition, double-layer finline, with two identical antipodal finlines located on the upper and lower finlines respectively, is also employed to realize the function of power divider. Therefore, a compact transition with the function of divider can be realized.

Due to the nonresonant configuration of this transition and the high symmetries of the double-layer finline and TE10 mode of the waveguide, the amplitudes and phase differences of two SIW branches should be identical, which decides the wideband power-dividing essence of this transition. However, the bandwidth of this transition suffers from the impedance matching between waveguide and the doublelayer finline, which will mainly decide the bandwidth of the transition.

In addition, the distance between the upper and lower finlines is also very important. A larger distance means more capacity in height, which is good for assembly of amplifier chip. However, too large distance makes it difficult to match waveguide with the double-layer finline. Therefore, the proper distance is a tradeoff of the two important factors. 


\subsection{Double-microstrip transition design}

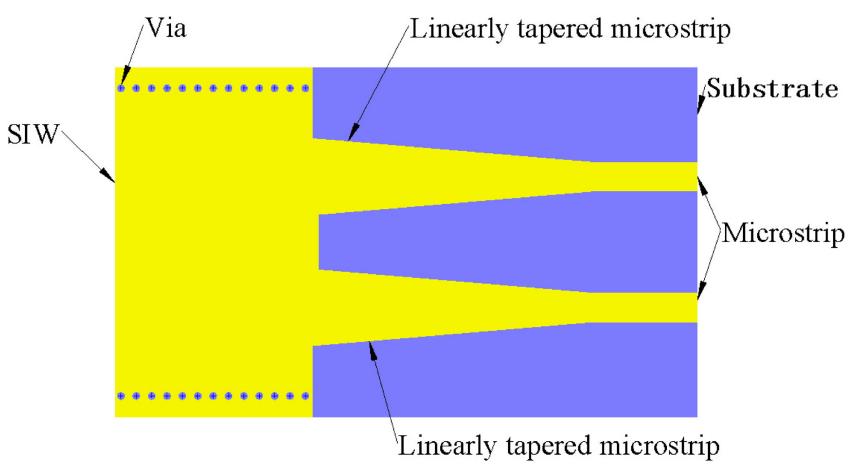

Fig. 3. Double-microstrip transition

For the microwave amplifier chip, microstrip is usually used as the input and output transmission line, which can easily be connected to amplifier using wire bonding process. Therefore, in order to be compatible with amplifier chip, SIW should be finally transited into microstrip [6,9, 10]. In [6], the linearly tapered transition between SIW and microstrip is widely used. For this transition, a tapered microstrip is inserted between SIW and microstrip, which can realize the mode transition of two different transmission lines, as well as match their impedances well. Like the double-layer finline [2], this transition can be developed into a new one (see Fig. 3), which can divide the input power into two identical ways by two identical tapered microstrip lines placed symmetrically. Depending on the high symmetries of TE10-like mode of SIW and two identical tapered microstrip lines, this transition also posseses wideband power-dividing essence. In addition, the spacing between the two microstrip lines is still a tradeoff of enough capacity for amplifier chip assembly and good impedance matching.

\section{Divider design}

The divider (see Fig. 1) consists of the two proposed transitions, and each SIW branch of the double-layer transition is cascaded to a double-microstrip transition. Thus the input power can be divided into four identical microstrip branches. In order to reduce the insertion loss of SIW section and improve the combing efficiency as power combiner, the length of SIW should be as short as possible.

For power-combining amplifier, the capability of heat sinking for amplifier chip is very important, which may limit the maximum output power of the amplifier chip. Therefore, heat sinking is also considered in the divider design. As shown in Fig. 1, the microstrips are configured face to face, and heat produced by the amplifier chips assembled on the microstrip can rapidly be transferred outward.

Due to the wideband essence of the two kinds of transitions, the bandwidth of the cascaded divider should be broad. Thus, a broadband divider based on SIW and double-layer finline can be designed.

\section{Simulated and experiment results}

For the proposed power divider, Rogers 5880 with a dielectric constant of 2.2 and a thickness of $0.254 \mathrm{~mm}$ was used as the substrate of SIW and microstrip line. The 


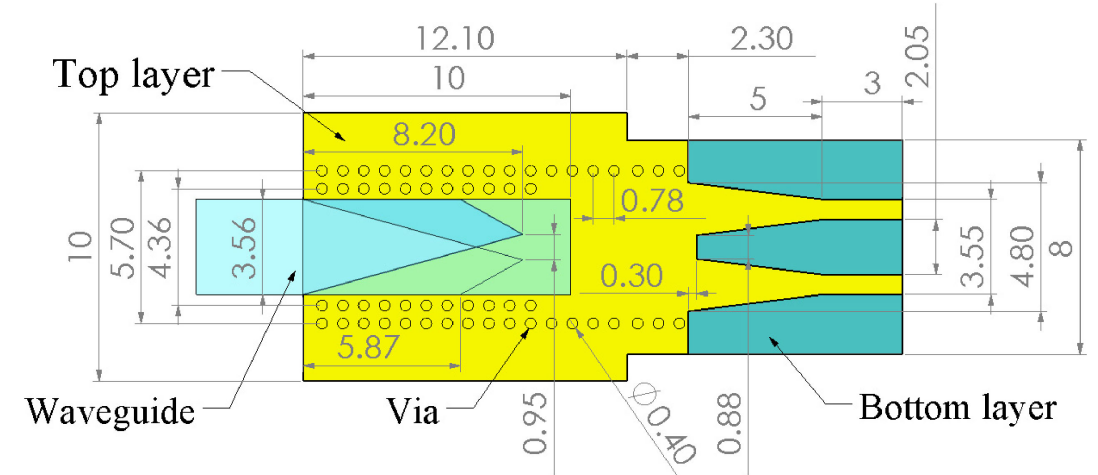

Fig. 4. The structure of the four-way divider

proposed transitions and divider were simulated and optimized in HFSS, and the structure of the divider with its dimensions is shown in Fig. 1 and Fig. 4. The S-parameters (see Fig. 5(a) and (b)) of the divider were also simulated.

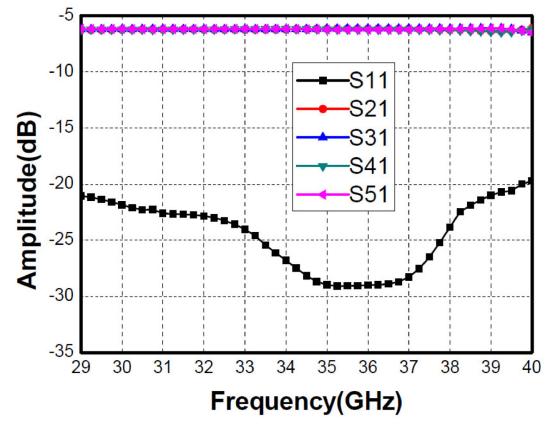

(a)

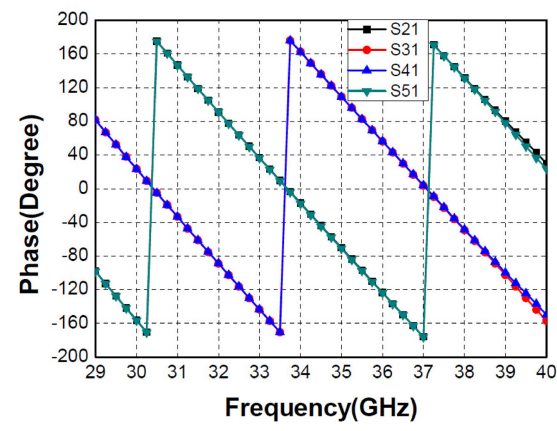

(b)

Fig. 5. Simulated results of the four-way power divider (a) Magnitude (b) Phase

As shown in Fig. 5(a), equal power division $(-6.2 \pm 0.2 \mathrm{~dB})$ is achieved at each microstrip port at $29-40 \mathrm{GHz}$, which is very close to the desired value of $-6 \mathrm{~dB}$. As shown in Fig. 5(b), the phase differences of two microstrip branches on the same substrate are uniform, and those on different finlines are 180 out of phase. A passive divider/combiner circuit was built by placing two identical dividing circuits back to back (Fig. 6), and the left and right parts of the power divider/ combiner also act as the role of heat sink respectively. As shown in Fig. 7, the measured results of the power divider/combiner show a good agreement with the simulated results. The measured minimum insertion loss of $1.3 \mathrm{~dB}$ is achieved with a return loss of $24 \mathrm{~dB}$ at $35.6 \mathrm{GHz}$, which indicates a maximum power-combining efficiency of $86 \%$ for the combining circuit, and the power-combining efficiency is higher than $75 \%$ at $29-40 \mathrm{GHz}$. The increased insertion loss compared with the simulated one is most likely due to the transmission losses of SIW and microstrip line and the mismatch of the amplitudes and phases of four microstrip branches. The increased reflection coefficient is mainly due to the fabrication and assembly errors. 


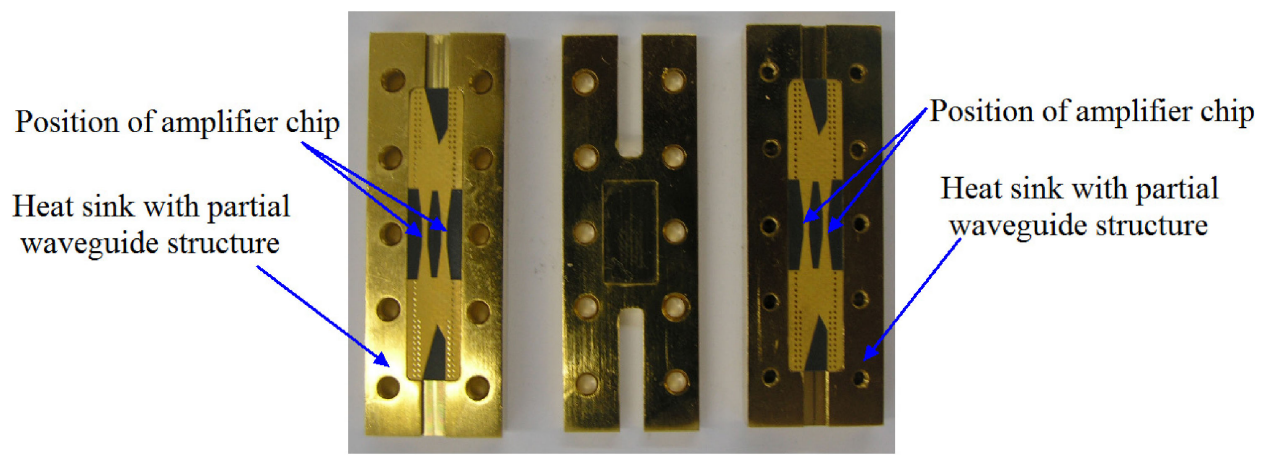

Fig. 6. The prototype of the proposed divider/combiner

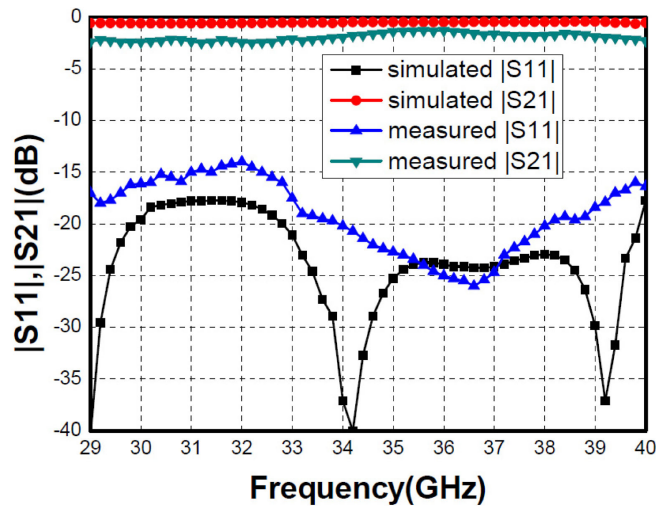

Fig. 7. The simulated and measured results of the proposed divider/ combiner placed back-to-back

\section{Conclusion}

A compact four-way power/combiner based on SIW and double-layer finline is designed and measured. As well as the compact structure, the proposed power divider/combiner is characterized by its wide bandwidth, high power-combining efficiency, ease of fabrication and assembly, and efficient heat sinking of amplifier chip.

\section{Acknowledgments}

This work was supported by the Fundamental Research Funds for the Central Universities of China (Grant No. ZYGX2012J030) and the Project-sponsored by OATF, UESTC. 